\title{
Dose Reduction in Computed Tomography of the Chest: Image Quality of Iterative Reconstructions at a 50\% Radiation Dose Compared to Filtered Back Projection at a $100 \%$ Radiation Dose
}

\author{
Dosisreduktion in der Thorax-CT: Vergleich der Bildqualität bei 50\% Dosis und \\ iterativer Bildrekonstruktion mit 100\% Dosis und gefilterter Rückprojektion
}

Authors

Affiliations
M. S. May' ${ }^{1}$, A. Eller ${ }^{1}$, C. Stahl' ${ }^{1}$, W. Wuest ${ }^{1}$, M. Scharf ${ }^{1}$, M. Hammon ${ }^{1}$, P. Dankerl ${ }^{1}$, P. M. Schlechtweg ${ }^{1}$, T. Allmendinger ${ }^{2}$, M. Sedlmair ${ }^{2}$, B. Schmidt ${ }^{2}$, M. Uder ${ }^{1}$, M. M Lell ${ }^{1}$

Department of Radiology, University Hospital Erlangen

${ }^{2}$ Healthcare, Siemens AG, Forchheim

\author{
Key words \\ Q thorax \\ - CT \\ technical aspects \\ - image manipulation/recon- \\ struction
}

received $\quad 15.2 .2013$

accepted $\quad 18.10 .2013$

Bibliography

DOI http://dx.doi.org/

10.1055/s-0033-1356254

Published online: 29.1.2014

Fortschr Röntgenstr 2014; 186: 576-584 ๑ Georg Thieme

Verlag KG Stuttgart · New York . ISSN 1438-9029

\section{Correspondence Dr. Matthias Stefan May Radiologisches Institut, Universitätsklinik Erlangen Maximiliansplatz 1 90154 Erlangen Tel.: ++49/9131/8536065 Fax: ++49/9131/8536068 matthias.may@uk-erlangen.de}

\section{Abstract \\ $\nabla$}

Purpose: The aim of this study was to evaluate the potential of iterative reconstruction (IR) in chest computed tomography (CT) to reduce radiation exposure. The qualitative and quantitative image quality of standard reconstructions with filtered back projection (FBP) and half dose (HD) chest CT data reconstructed with FBP and IR was assessed.

Materials and Methods: 52 consecutive patients underwent contrast-enhanced chest CT on a dual-source CT system at $120 \mathrm{kV}$ and automatic exposure control. The tube current was equally split on both tube detector systems. For the HD datasets, only data from one tube detector system was utilized. Thus, FD and HD data was available for each patient with a single scan. Three datasets were reconstructed from the raw data: standard full dose (FD) images applying FBP which served as a reference, HD images applying FBP and IR. Objective image quality analysis was performed by measuring the image noise in tissue and air. The subjective image quality was evaluated by 2 radiologists according to European guidelines. Additional assessment of artifacts, lesion conspicuity and edge sharpness was performed.

Results: Image noise did not differ significantly between HD-IR and FD-FBP $(\mathrm{p}=0.254)$ but increased substantially in HD-FBP $(\mathrm{p}<0.001)$. No statistically significant differences were found for the reproduction of anatomical and pathological structures between HD-IR and FD-FBP, subsegmental bronchi and bronchioli. The image quality of HD-FBP was rated inferior because of increased noise.

Conclusion: A $50 \%$ dose reduction in contrast-enhanced chest CT is feasible without a loss of diagnostic confidence if IR is used for image data reconstruction. Iterative recon- struction is another powerful tool to reduce radiation exposure and can be combined with other dose-saving techniques.

Key Points:

- Iterative reconstructions allow for image noise and artifact reduction.

- Comparable image data can thus be attained even at $50 \%$ radiation dose.

- Diagnostic confidence remains unaffected. Citation Format:

- May MS, Eller A, Stahl C et al. Dose Reduction in Computed Tomography of the Chest: Image Quality of Iterative Reconstructions at a 50\% Radiation Dose Compared to Filtered Back Projection at a $100 \%$ Radiation Dose. Fortschr Röntgenstr 2014; 186: 576-584

\section{Zusammenfassung}

$\nabla$

Ziel: Evaluation der iterativen Rekonstruktionstechnik (IR) zur Dosisreduktion in der ThoraxCT. Vergleich quantitativer und qualitativer Kriterien zur Bildqualität bei um $50 \%$ reduzierter Dosis (HD) mit den mittels gefilterter Rückprojektion (FBP) rekonstruierten Datensätzen bei voller (FD) und HD.

Material und Methode: Bei 52 konsekutiven Patienten wurde der anatomisch modulierte Röhrenstrom bei $120 \mathrm{kV}$ zu gleichen Teilen auf die zwei Röhren-Detektor-Einheiten eines Dual-Source-CT aufgeteilt. Aus jeder Untersuchung wurden je $3 \mathrm{Da}-$ tensätze rekonstruiert: FD-FBP durch Auswertung der Rohdaten aus beiden, sowie HD-FBP und HDIR aus Rohdaten eines einzelnen Röhren-DetektorSystems. Die objektive Bewertung der Bildqualität erfolgte durch Messung des Bildrauschens, die subjektive durch 2 Untersucher analog europäischer Richtlinien. Zusätzlich wurden Bildartefakte sowie die Abgrenzbarkeit und Randschärfe von Läsionen evaluiert. 
Ergebnisse: Das Bildrauschen in HD-IR und FD-FBP zeigte keine signifikanten Unterschiede ( $\mathrm{p}=0,254)$ und war in HD-FBP deutlich erhöht $(p<0,001)$. Mit Ausnahme der Subsegmentbronchien und Bronchiolen zeigten FD-FBP und HD-IR sowohl bzgl. der scharfen Darstellung pulmonaler und mediastinaler Strukturen als auch der pathologischen Läsionen keine signifikanten Unterschiede. Die HD-FBP-Bilddaten wurden hingegen überwiegend qualitativ signifikant schlechter bewertet.

Schlussfolgerung: Bei der Kontrastmittel-CT des Thorax kann eine vergleichbare Bildqualität und diagnostische Wertigkeit bei halber Strahlendosis mittels IR dank Rausch- und Artefaktreduktion erreicht werden. Iterative Rekonstruktionen ermöglichen eine deutliche Verringerung der CT-Strahlendosis und können mit anderen Reduktionsalgorithmen kombiniert werden.

\section{Introduction}

$\nabla$

The improvement of the spatial and temporal resolution of multidetector computed tomography (MDCT) resulted in a significant increase in the indication for the method and thus an increase in the cumulative radiation exposure [1 3]. In a European comparison, the greatest number of X-ray examinations per capita with the consequently highest radiation exposure per inhabitant and per year is performed in Germany (effective dose, $E D=1.9 \mathrm{mSv} / \mathrm{a}$ ) [4]. Therefore, radiation exposure reduction has become a central topic in diagnostic radiology [5]. Since the thorax is the third most common region for CT examination in Germany and represents the second highest collective dose [4], even minimal dose reductions in the individual examinations affect the collective ED. While the use of lead and bismuth shields is controversial [6 - 8], the use of anatomy-based dose modulation is established and has been substantiated by a number of studies [9, 10]. Adaptive z-collimation [11] can effectively reduce the over-ranging that becomes an issue with an increasing detector panel width. It was able to be shown in numerous phantom-based and follow-up studies that the image quality in chest $\mathrm{CT}$ can be improved by iterative reconstruction algorithms with acceptable reconstruction times $[12,13]$ resulting in the potential for dose reduction [1416]. In iterative reconstruction (IR), an output image is initially calculated from the measured projection data. From this image a comparison dataset is then calculated via a forward projection and is compared to the output data. Depending on the type of algorithm, this is performed separately in the image data room or with inclusion of the raw data room. If the results deviate from one another, a correction image is calculated. Data is then again synthesized from this correction image and compared to the measured data. This iteration loop is repeated until a defined termination criterion is fulfilled. To establish IR at a reduced radiation dose for patients, the goal of this study was to compare the image quality of standard reconstruction (filtered back projection, FBP) at a full dose (FD) to the image quality of an image-based iterative reconstruction at a half dose (HD) in a single-phase and intraindividual study design (non-inferiority study) and to evaluate clinically relevant lesions in addition to the already published parameters.

\section{Materials and Methods}

\section{$\nabla$}

\section{Patients}

52 consecutive patients with a clinical indication for contrast-enhanced chest CT were prospectively examined on a 128-row dual-source (DS) unit (Somatom Definition Flash, Siemens Healthcare, Forchheim, Germany). The most common indication was tumor staging $(n=42)$ followed by dyspnea of an unclear origin $(n=6)$ and the search for an infectious focus $(n=4) .38$ men and 14 women with an average age of $62 \pm 13$ years and a body mass index of $26 \pm 5 \mathrm{~kg} / \mathrm{m}^{2}$ were included. The exclusion criteria were known intolerance to contrast agents, renal insufficiency (eGFR $<60 \mathrm{ml} /$ $\min / 1.73 \mathrm{~m}^{2}$ ), hyperthyroidism (TSH $<0.3 \mathrm{mU} / \mathrm{l}$ ) and BMI $>40 \mathrm{~kg} / \mathrm{m}^{2}$. The last criterion was selected to rule out incomplete exposure by the smaller of the two tube-detector systems (field of measurement $(F O M)=33 \mathrm{~cm}$ ). Written informed consent after clarification by the physician was available for all patients and the study was approved by the ethics commission.

\section{CT technique}

All examinations were performed with a tube voltage of $120 \mathrm{kV}$, a referenced tube current time product of $100 \mathrm{mAs}$ with real-time tube current modulation (CareDose $4 \mathrm{D}$, Siemens Healthcare, Forchheim, Germany), a rotation time of $0.5 \mathrm{~s}$, a pitch of 0.8 , and a total collimation of $38.4 \mathrm{~mm}$ $(64 \times 0.6 \mathrm{~mm})$ using a $\mathrm{z}$-spring focus. The calculated tube current was divided equally between the two X-ray tubes. All patients received $0.4 \mathrm{~g}$ of iopromide (Ultravist $370^{\circledR}$, Bayer Healthcare, Berlin, Germany) per kg bodyweight with an automatic 2-piston injector (Stellant D CT, Medrad Inc., Warrendale, PA, USA) and a flow rate of $3 \mathrm{ml} / \mathrm{s}$ followed by $50 \mathrm{ml}$ of $0.9 \%$ saline solution administered intravenously via a peripheral indwelling venous cannula. The scan delay was $50 \mathrm{~s}$.

\section{Dose calculation}

The radiation exposure was estimated from the dose protocol on the basis of the volume CT dose index (CTDI $\left.{ }_{\text {vol }}\right)$ and the dose length product (DLP). The effective dose was calculated from the DLP via the conversion factor for chest CT $\left(\mathrm{k}=0.0145 \mathrm{mSv} / \mathrm{mGy}^{*} \mathrm{~cm}\right)$ [17].

\section{Image reconstruction}

The separate and simultaneous reading out of the data from the two detector systems makes it possible to calculate an image dataset with a half dose (HD) as well as a full dose (FD) in a single examination without an additional radiation dose for the patient. The reference was the FD-FBP datasets to which the HD-IR and HD-BP images were compared ( $\bullet$ Fig. 1). Each raw dataset was calculated with a high-resolution (B70/I70) and a soft (B40/I40) convolution kernel with a slice thickness of $5 \mathrm{~mm}$ and $1 \mathrm{~mm}$ and a reconstruction increment of $5 \mathrm{~mm}$ and $0.7 \mathrm{~mm}$, respectively. IR was performed using the IRIS algorithm (iterative reconstruction in image space, Siemens Healthcare, Forchheim, Germany). 5 iteration steps were performed for the calculation of each HD-IR dataset in this study. The duration of the thick-slice reconstructions was recorded for the individual datasets. 


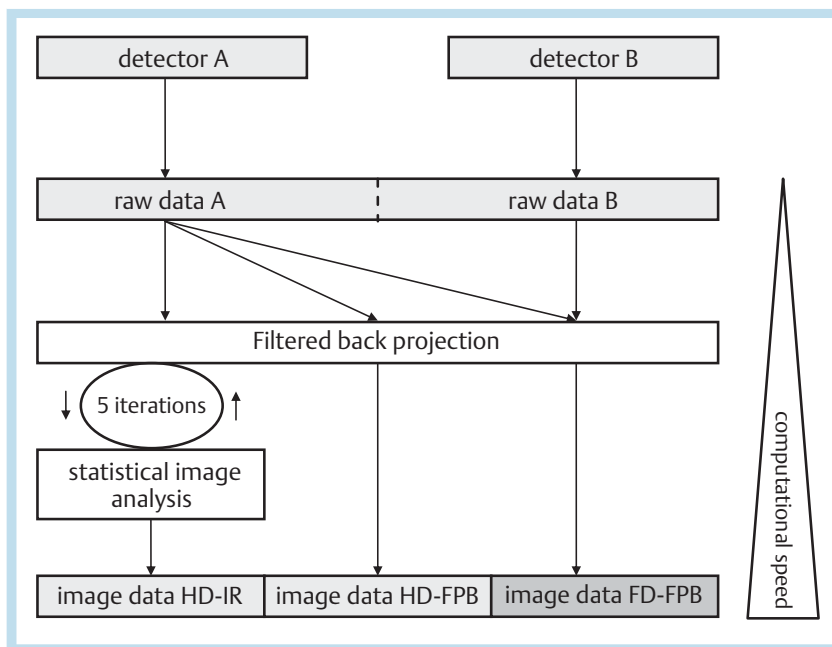

Fig. 1 Flowchart of image reconstruction: Only detector A is read out for half-dose image reconstructions. Iterative reconstructions repetitively compare image aspects such as edge information and image noise with the output image calculated via filtered back projection.

\section{Image quality}

After anonymization and removal of all acquisition and reconstruction parameters, all image series were evaluated on a 3 D workstation (Syngo MMWP VE36A, Siemens Healthcare, Erlangen, Germany). The objective image quality was measured at the height of the maximum extension of both heart chambers as a standard deviation of the attenuation values of round measurement areas in the left and right ventricle, the descending aorta, the autochthonous back musculature on the right and left of the spinal column and in the air ventral to the thorax ( $\bullet$ Fig.2). The measurement areas were placed in an identical position in all 3 datasets via the copy function while avoiding artifacts. The subjective image quality was evaluated by two radiologists ( 14 and 4 years professional experience) in accordance with the European Guidelines on Quality Criteria for Computed Tomography [18]. The lung parenchyma was evaluated in the sharp convolution kernel with window values of 1700/-600 (width/center) and the mediastinal structures were evaluated in the soft convolution kernel at 400/40 (width/center). The complete image of the thoracic wall, thoracic aorta, vena cava, intrathoracic vessels, heart, and lung parenchyma and the sharp visualization of 11 mediastinal structures (front mediastinum, trachea, main bronchi, paratracheal tissue, lymph nodes, esophagus, heart, aortic contour, aortic wall, superior vena cava, inferior vena cava, other mediastinal vessels) and 9 pulmonary structures (costal pleura, mediastinal pleura, lung fissures, segmental pulmonary vessels, subsegmental vessels, segmental bronchi, subsegmental bronchi and bronchioles, secondary lung lobules, lung parenchyma) were evaluated on the basis of a dichotomous Likert scale ( 1 -completely or clearly visualized, 2 - incompletely or unclearly visualized) [18]. General image aspects such as subjective image noise and spatial resolution were evaluated on a 3-point scale (1 too low, 2 - optimal, 3 - too high), and the general diagnostic applicability was evaluated using a 4-point Likert scale (1 highly acceptable, 2 - minimally acceptable, 3 - acceptable with restrictions, 4 - unacceptable) [18]. The same scale was used for additional evaluation of pathological lesions with re-

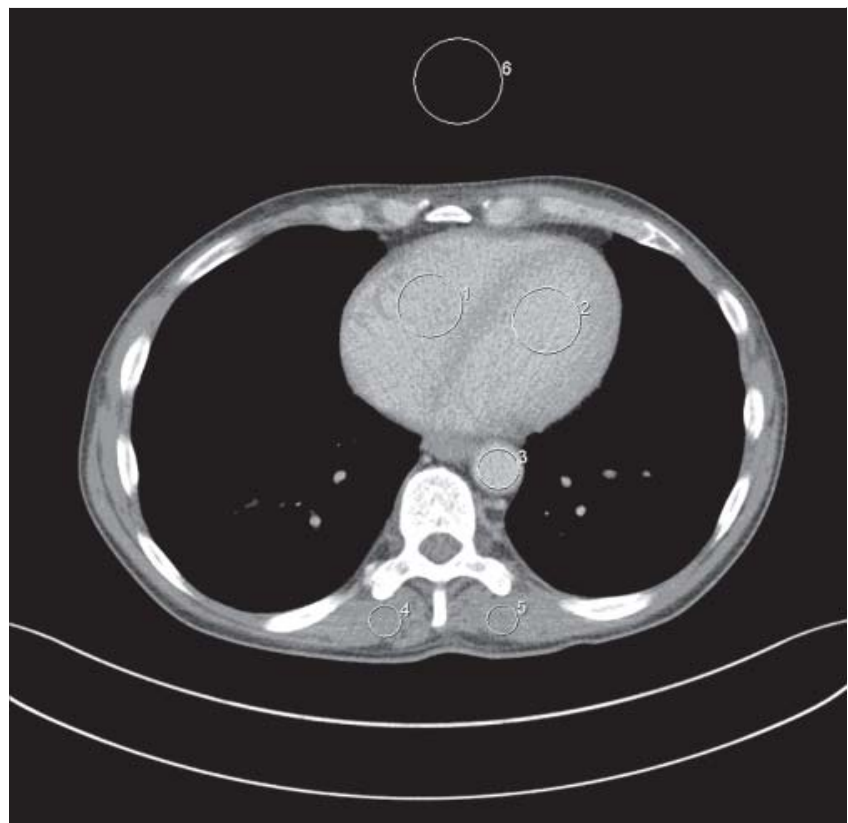

Fig. 2 Objective data analysis via voxel evaluation in the right and left ventricle, in the descending aorta, bilateral in the erector spinae and in air.

spect to contrast and edge sharpness. In the event of multiple lesions of one entity, the smallest was always used for evaluation. Moreover, image artifacts (spiral artifacts, streak artifacts, truncation artifacts, and a coarse image appearance) were also evaluated according to a 4-point scale (1 - no artifact, 2 - artifact with moderate effect on the visualization of a structure, 3 - artifact with negative effect on the visualization of a structure, 4 - artifact impairing diagnosis).

\section{Statistics}

After inclusion of the first 16 patients, a case number estimation for an equivalence hypothesis (non-inferiority) of the HD-IR data compared to the FD-FBP data was performed on the basis of a preliminary evaluation of the quantitative image quality with a statistical power of $80 \%$ [19]. All results are given as mean \pm standard deviation (SD) for continuous variables and as percentages and ranges for categorical variables. The equality of normally distributed, interval-scaled data was checked via one-way analysis of variance (ANOVA) and then compared pair-wise via post-hoc tests, via Tamhane's T2 test in the case of inequality of the variances in Levene's test and via the Bonferroni test in the case of variance equality. Since the ordinal-scaled data of the qualitative image analysis were not distributed normally, the non-parametric Friedman test and then a post-hoc test according to Schaich were used $[20,21]$. The agreement between the examiners was checked for the categorical parameters by the Cohen's Kappa test. The significance level was defined as $<0.05$. The total statistical evaluation was performed with the software PASW Statistics 18.0 (SPSS Inc., Chicago, IL).

\section{Results}

To rule out inferiority of the HD-IR data in the $90 \%$ confidence interval with $80 \%$ reliability, a case number of 50 pa- 
Table 1 Mean size [pixels] and density values [HU] of the regions of measurement.

\begin{tabular}{|lllccccc} 
& descending aorta & right ventricle & left ventricle & musculature right & musculature left & air \\
\hline Field of measurement [pixels] & 383 & 987 & 1244 & 171 & 205 & 2722 \\
\hline FD-FBP [HU] & 137 & 129 & 128 & 55 & 58 & -999 \\
\hline HD-IR [HU] & 137 & 133 & 130 & 53 & 54 & -998 \\
\hline HD-FBP [HU] & 138 & 133 & 131 & 53 & 0.54 & 0.43 & -999 \\
\hline p-value & 0.99 & 0.62 & 0.32 & 0.39 &
\end{tabular}

tients at a maximum allowed image noise deviation of $10 \%$ was calculated.

The average examination length ( $\mathrm{z}$-axis) was $38 \pm 4 \mathrm{~cm}$ with an average examination time of $6.2 \pm 0.6 \mathrm{~s}$. The anatomically controlled tube current modulation resulted in $82 \pm 36$ effective mAs (range: 33 - 236 eff. mAs), corresponding to approx 41 eff. mAs per X-ray tube. Therefore, the median values were: FD-CTDI 6.2 mGy (range: 2.9 - 20.2 mGy), FD-DLP $245 \mathrm{mGy}^{*} \mathrm{~cm}$ (range: $111-858 \mathrm{mGy}^{*} \mathrm{~cm}$ ) and FD-ED $3.6 \mathrm{mSv}$ (range: $1.6-12.4 \mathrm{mSv}$ ). The reconstruction of a chest image series $(5 / 5)$ took $12 \pm 0.4 \mathrm{~s}$, corresponding to an average reconstruction speed of 6.3 images/s, for the FBP algorithm and $55 \pm 2.1 \mathrm{~s}$, corresponding to 1.3 images/s, for IR. This results in a reconstruction speed that is 4.7 times faster for FBP than for IR. The selection of the filter kernel did not affect the reconstruction speeds.

\section{Objective image quality}

A balanced vascular contrast of approx. $130 \mathrm{HU}$ was generated with the selected contrast agent protocol. The average density values did not show any significant differences in the various image reconstructions ( $\bullet$ Table 1 ). As expected, an increase in image noise in the intracorporeal measurement regions of approx. $40 \%$ was observed in the HD-FBP reconstructions compared to HD-IR and FD-FBP. With an increase of 34.8, the noise measurement in air is lower compared to FD-FBP due to the limited density scale at -1024 HU and the associated shift of the distribution curve of the measured values. In contrast, the differences in the measured image noise in the full-dose and IR datasets were not significant $(p=0.25)$. The average image noise in HD-IR was $-1.3 \%$ less than in FD-FBP ( $\bullet$ Table 2 ).

\section{Subjective image quality}

Using the evaluated categorical parameters, both examiners achieved an average agreement of $\mathrm{K}=0.493$. With $\mathrm{K}=0.592$ the value for the FD dataset was highest followed by HD-IR $(\mathrm{K}=0.483)$ and HD-FBP $(\mathrm{K}=0.425)$. According to Landis and Koch, there is moderate agreement for all 3 datasets [22].

\section{Image impression}

The evaluation of the three datasets by both examiners resulted in statistically significantly better values for FD-FBP and HD-IR $(\mathrm{p}<0.001)$ compared to HD-FBP with respect to the general diagnostic value. The differences between FDFBP and HD-IR were not significant. Of the 52 datasets, 30 (58\%) were evaluated as highly acceptable, 21 (40\%) as minimally acceptable, and $1(2 \%)$ as acceptable with restrictions after image reconstruction with HD-FBP. In contrast, only one IR dataset and one FD dataset ( $2 \%$ ) were evaluated as minimally acceptable while the rest were evaluated as highly acceptable $(n=51 ; 98 \%)$. The subjective image noise
Table 2 Image noise $[\mathrm{HU}]$ and deviation from FD-FBP as percentage.

\begin{tabular}{|llll|}
\hline field of measurement & FD-FBP & HD-IR & HD-FBP \\
\hline descending aorta & 20.9 & $19.5(-6.7 \%)$ & $30.6(+46.2 \%)$ \\
\hline right ventricle & 20.5 & $19.4(-5.1 \%)$ & $29.6(+44.4 \%)$ \\
\hline left ventricle & 21.6 & $21.2(-1.9 \%)$ & $30.2(+40.1 \%)$ \\
\hline right musculature & 20.2 & $20.4(+0.7 \%)$ & $28.9(+43.2 \%)$ \\
\hline left musculature & 20.7 & $20.4(-1.4 \%)$ & $28.8(+39 \%)$ \\
\hline air & 10.2 & $10.8(+6.7 \%)$ & $13.7(+34.8 \%)$ \\
\hline
\end{tabular}

was evaluated as too high in $98 \%(n=51)$ of the HD-FBP datasets and in only $4 \%(\mathrm{n}=2)$ of the FD-FBP and HD-IR datasets. Insufficient noise was not found. Only the differences between HD-FBP and FD-FBP/HD-IR were significant. The achieved spatial resolution was classified as optimal for all sectional image series of the 3 datasets.

\section{Anatomy}

It was possible to completely visualize the thoracic wall, thoracic aorta, vena cava, heart, lung parenchyma, and contrast-filled vessels in all three datasets of the patient collective. The FD-FBP and HD-IR datasets did not show any significant differences in the visualization of mediastinal structures $(\mathrm{p}=0.865)$ and pulmonary structures $(\mathrm{p}=0.141)$. A loss of quality was only seen in the depiction of the subsegmental bronchi and bronchioles in the HD-IR datasets in the individual comparison $(\mathrm{p}=0.008)$. However, the sharp visualization of both the mediastinal and the pulmonary structures was significantly worse in HD-FBP than in HDIR and FD-FBP ( $\mathrm{p} \leq 0.018$ ). In the individual comparison, significant differences in the mediastinal vascular visualization (inferior vena cava, heart, aortic contour and wall) were described. The depiction of the lungs showed significant advantages for the comparison between FD-FBP and HD-FBP with regard to the majority of HR structures (subsegmental vessels, subsegmental bronchi and bronchioles, secondary lung lobules, and lung parenchyma) and for the comparison between HD-IR and HD-FBP with regard to the depiction of the lung parenchyma ( $\bullet$ Table 3 ).

\section{Lesions}

Overall, 141 pathological changes, 93 in the lung parenchyma ( $\odot$ Fig. 3, 4) and 48 in the scanned soft tissues ( $\bullet$ Fig. 5, 6), were documented in 52 patients. 43 (30\%) were classified as malignant and 98 (70\%) as benign. For the evaluation of the subjective image quality, these were classified on the basis of the different underlying morphologies as changes in the lung framework $(n=38)$, pulmonary circular foci $(n=55)$, vascular changes $(n=15)$, and soft tissue lesions $(n=33)$. The lesions were between 1 and $44 \mathrm{~mm}$ in size (average value $7 \mathrm{~mm}$ ). While no significant differences in 

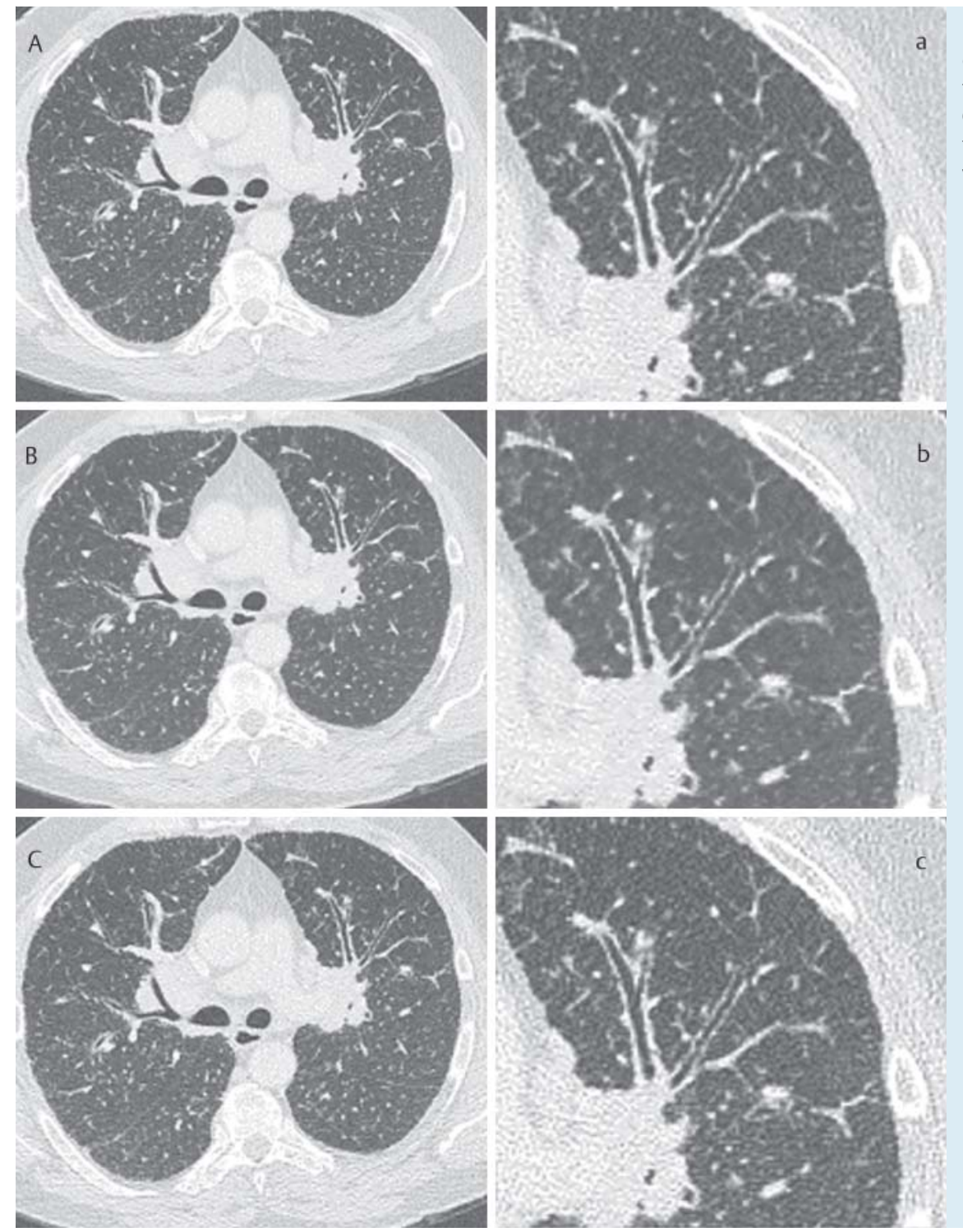

Fig. 3 46-year-old male $\left(B M I=28 \mathrm{~kg} / \mathrm{m}^{2}\right)$ with dyspnea. 1-mm slice thickness and sharp convolution kernel for FD-FBP A/a, HD-IR B/b und HD-FBP C/c. Centrilobular nodules, consolidations and thickening of the bronchial walls in sarcoidosis are visible in all 3 reconstructions.

Table 3 P-values of the statistically significant post-hoc tests. Non-significant p-values of the subgroups as well as anatomical structures and lesions without significant results in the Friedman test are not shown.

\begin{tabular}{|c|c|c|c|}
\hline & \multicolumn{3}{|l|}{ post-hoc } \\
\hline & $\begin{array}{l}\text { FD-FBP vs. } \\
\text { HD-IR }\end{array}$ & $\begin{array}{l}\text { FD-FBP vs. } \\
\text { HD-FBP }\end{array}$ & $\begin{array}{l}\text { HD-FBP vs. } \\
\text { HD-IR }\end{array}$ \\
\hline mediastinal anatomy & 0.865 & $<0.001$ & $<0.001$ \\
\hline heart & & 0.012 & 0.005 \\
\hline aortic contour & & 0.002 & 0.005 \\
\hline aortic wall & & 0.008 & 0.012 \\
\hline inferior vena cava & & 0.039 & \\
\hline pulmonary anatomy & 0.141 & $<0.001$ & 0.018 \\
\hline subsegmental vessels & & 0.039 & \\
\hline $\begin{array}{l}\text { subsegmental bronchi and } \\
\text { bronchioles }\end{array}$ & 0.008 & 0.001 & \\
\hline secondary lung lobules & & 0.039 & \\
\hline lung parenchyma & & 0.05 & 0.05 \\
\hline contrast of the lesions & 0.783 & 0.054 & 0.099 \\
\hline marginal definition of the lesions & 0.855 & 0.001 & 0.002 \\
\hline pulmonary circular foci & & 0.022 & 0.045 \\
\hline soft tissue lesions & & 0.005 & 0.007 \\
\hline
\end{tabular}

the contrast of the lesions with respect to the surrounding tissue could be shown in the three datasets in the individual comparison, the HD-FBP reconstruction showed statistically significant edge enhancement disadvantages with regard to intrapulmonary circular foci and soft tissue lesions. The statistically significant differences in the edge sharpness of lung framework changes in all three datasets in the Friedman test $(p=0.011)$ could not be confirmed in the pairwise post-hoc tests ( $\bullet$ Table 3 ).

\section{Artifacts}

Streak artifacts occurring primarily at high-contrast tissue boundaries, e.g. the heart $(n=35)$, the erector spinae $(n=27)$, the ribs $(n=8)$, and around the head of the humerus ( $n=6)$, were detected most frequently. They occurred significantly more frequently in HD-FBP than in FD-FBP $(p<0.001)$ and HD-IR $(p<0.001)$. They affected the image quality of the HD-FBP images in 29 cases. This was not the case in the remaining datasets $(p=0.492)$. Diagnosis-limiting artifacts were not observed. With respect to the image structure, a pixilated appearance of the HD-IR reconstructions with a moderate effect on the visualization of the 

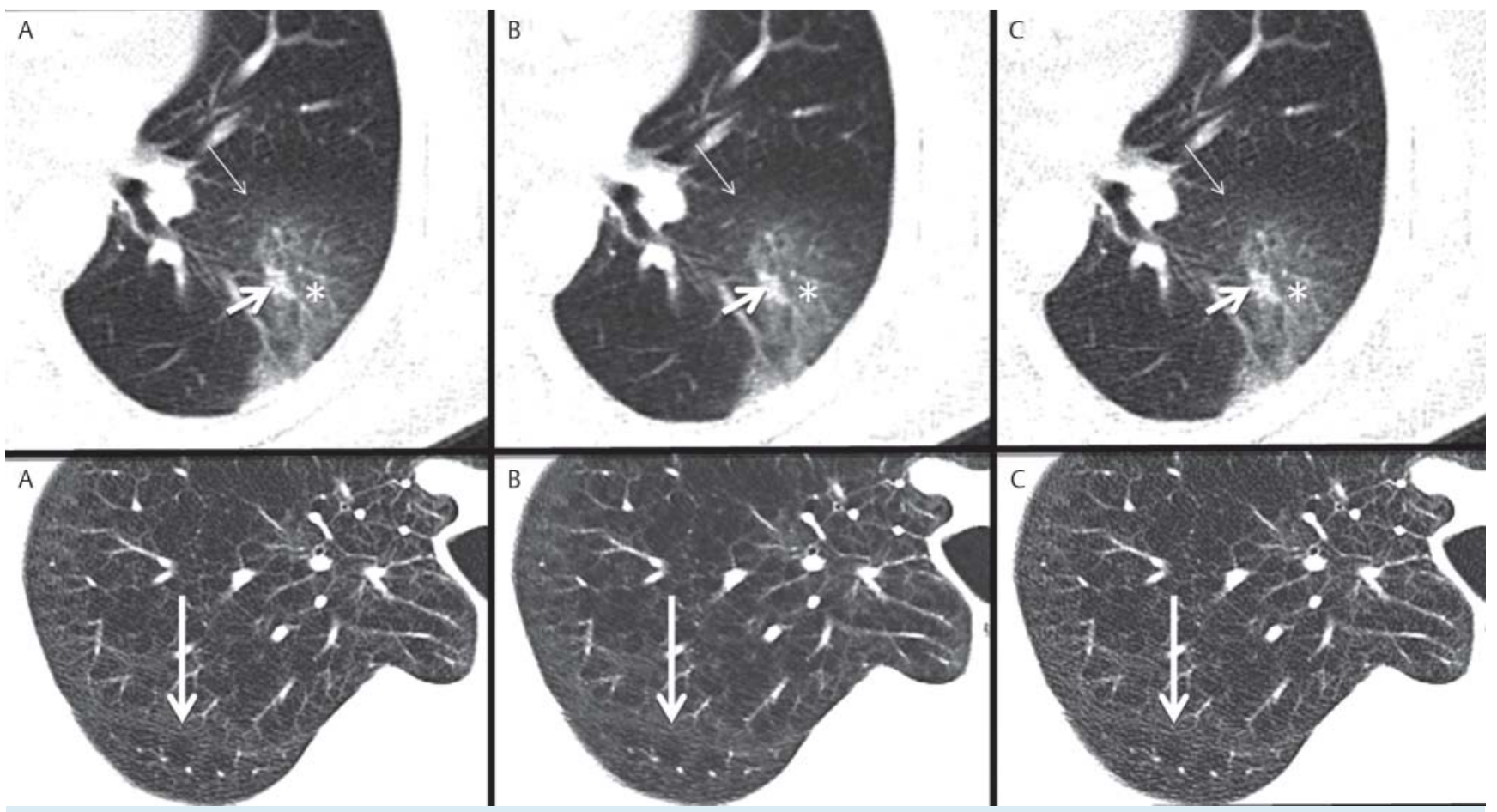

Fig. 4 Top: 65 -year-old male $\left(B M I=24 \mathrm{~kg} / \mathrm{m}^{2}\right)$ with fever and dyspnea. 5 $\mathrm{mm}$ slice thickness and high-resolution reconstruction kernel for FD-FBP A, HD-IR B and HD-FBP C. Pneumonia: consolidations (short arrow) and central parts of the ground-glass opacity are visible in all three reconstructions. However, peripheral expansion of the ground-glass opacity (long arrow) is

hardly distinguishable from the background image noise in HD-FBP. Bottom: 56 -year-old male $\left(B M I=17 \mathrm{~kg} / \mathrm{m}^{2}\right)$, follow-up for oral squamous cell carcinoma. Centrilobular emphysema of the lungs: Distinction between residual lung parenchyma and bullae (arrow) is limited in HD-FBP (C) due to the increased noise pattern and similar in HD-IR (B) and FD-FBP (A).

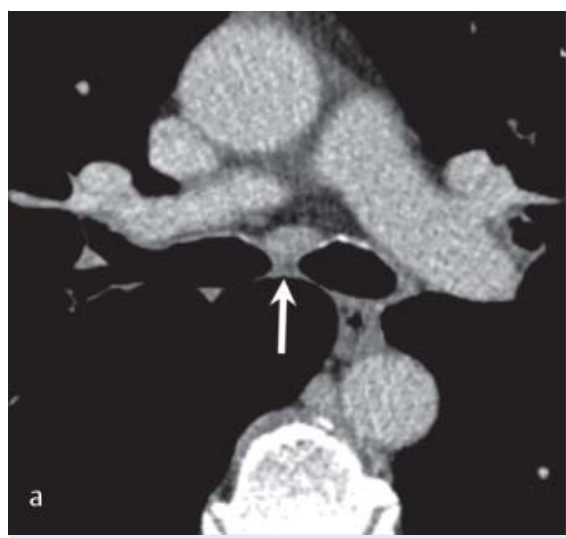

Fig. 5 49-year-old male (BMI $=24 \mathrm{~kg} / \mathrm{m} 2)$ with known bronchial carcinoma. 5-mm slice thickness and soft convolution kernel for reconstruction. Mediastinal lymph node metastasis (white arrow): The edge sharpness in
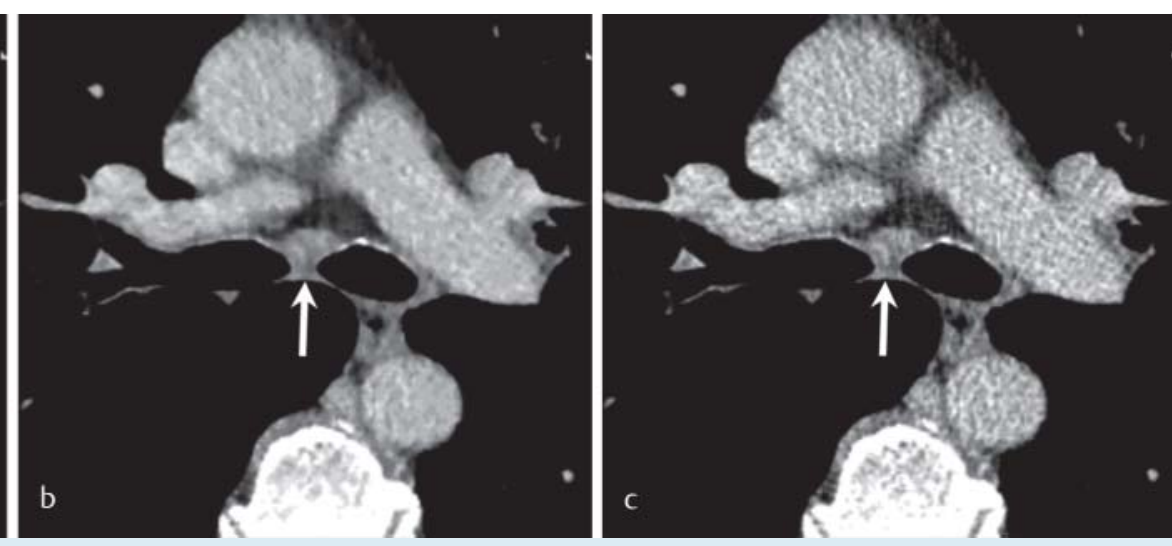

FD-FBP $\mathbf{A}$ and HD-IR $\mathbf{B}$ is superior to that of HD-FBP $\mathbf{C}$, while the lesion contrast is similar in all three datasets. structures was seen in 24 patients (46\%). This was uniformly distributed across the entire volume dataset and was not considered to negatively affect diagnosis. Distribution curves of the frequency spectrum of the image noise in the liver parenchyma calculated as an example show a reduced noise-power spectrum ( $\bullet$ Fig. 7 ) here as a verifiable correlate, as also published in the literature on the basis of a phantom model [23]. This did not occur in the FBP reconstructions. Spiral or truncation artifacts were not observed.

\section{Discussion}

$\nabla$

Without negatively affecting attenuation values and contrast, a constant image noise can be achieved via iterative image reconstruction even at a half dose in relation to reconstructions via filtered back projection. In contrast to HD-FBP datasets, an image quality comparable to that of FD-FBP could be achieved with IR. In contrast to HD-FBP, streak artifacts were significantly reduced with IR, and in contrast to FD-FBP, the noise texture was changed to a coarse image appearance and disadvantages in the depiction of the subsegmental bronchi and bronchioles were found. 

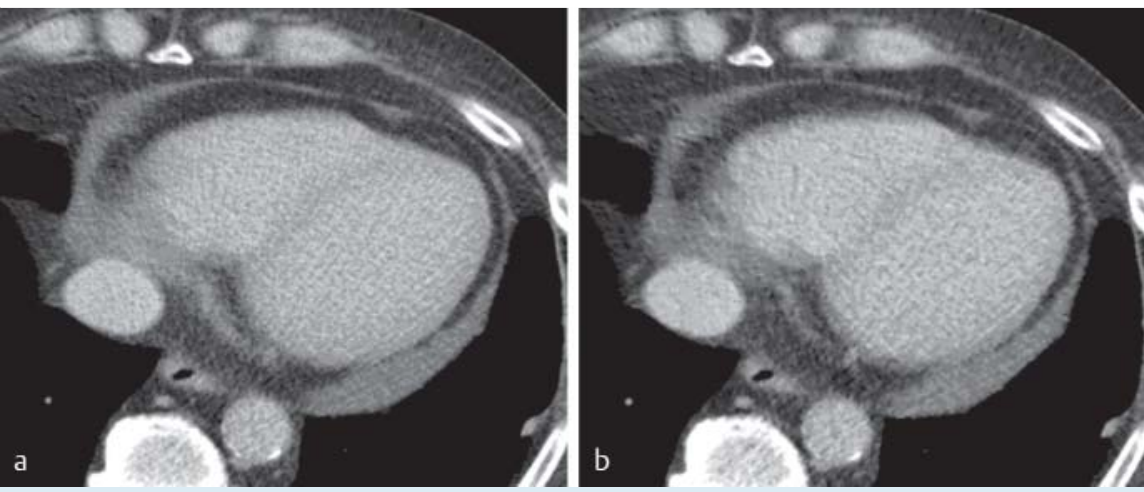

Fig. 6 65-year-old male $(B M I=27 \mathrm{~kg} / \mathrm{m} 2)$ for staging of cholangiocellular carcinoma. 5-mm slice thickness and soft convolution kernel for reconstruction. Pericardial effusion: The contour sharpness in FD-FBP a and HD-IR b is superior to that of HD-FBP c, while the contrast to the surrounding tissue is similar in all three datasets.

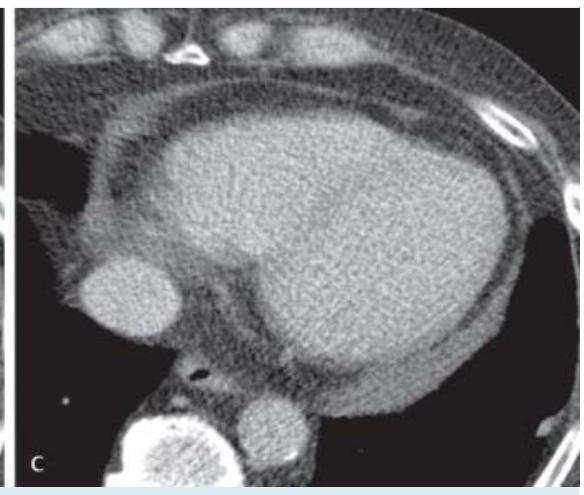

Due to the need for high-performance computers, iterative reconstructions were not compatible with the clinical routine for a long time. The steadily increasing capacity of processors and the development of new algorithms with shifting of the iterative correction loops from the raw data room to the image data room greatly increased the application area [24, 25]. There are currently different algorithms for iterative reconstruction that allow correction of artifacts in some cases via access to the raw data [26]. Either an improvement in image quality at an equivalent dose or constant image quality at a lower dose could be shown for different issues and examination regions [27-29]. However, the image-based algorithm examined in the present study allows calculation of iterative datasets regardless of the raw data and can therefore be used regardless of the time of acquisition for already archived image data. In a phantom study, Ghetti et al. were able to show a reduction of both the nominal image noise and the noise power spectrum while maintaining the spatial resolution [23]. Pontana et al. reported advantages with respect to the subjective image appearance and the objective image quality at a $30 \%$ dose reduction compared to the standard dose and FBP with a prototype of the IRIS algorithm in a follow-up study design using five iteration steps [14]. Hwang et al. had a comparable study approach and found the objective image quality at a half dose and using IRIS to be slightly worse compared to FD-FBP with respect to the edge sharpness of mediastinal structures [30], while the subjective image quality was increased [31]. The present study also shows slight advantages of HD-IR compared to the reference dose and FBP with respect to the anatomical structures on the basis of standardized evaluation criteria (European Commission on Radiological Protection). However, these are largely without statistical significance in contrast to HD-FBP. The additional evaluation of pathological changes makes it possible to check clinically relevant image aspects in the single-phase, intraindividual comparison with elimination of evaluation distortion due to recall bias, change in the orientation in the room, and morphological changes in the time course.

In detail, the image noise of IR at a half dose showed no statistical significance with respect to conventional image data at a full dose. In contrast, as expected, the FBP reconstructions followed the physical dependence of image noise on the radiation dose at a constant tube voltage by the factor $\sqrt{2}$. The iterative reconstruction algorithm did not affect the density values. Therefore, the nominal image contrast is maintained. The global evaluation of the datasets did not yield any statistically significant differences in the subjective image quality between FD-FBP and HD-IR. In contrast, HD- 
FBP was inferior in this regard. In the detailed examination of the anatomical structures, FD-FBP and HD-IR are equally superior to HD-FBP with respect to the depiction of mediastinal soft tissues and the general visualization of the lung. In contrast, the differences in the visualization of the HR structures are significantly smaller between the three datasets with advantages for HD-IR compared to HD-FBP and disadvantages compared to FD-FBP. In the comparison of FD-FBP and HD-IR, this is only significant in the evaluation of the subsegmental bronchi and bronchioles.

HD-IR reconstructions achieve image quality that is comparable to that of FD-FBP and superior to that of HD-FBP even with respect to the edge sharpness of intrapulmonary circular foci and soft tissue lesions as well as to the severity of beam-hardening artifacts.

Disadvantages of HD datasets compared to FD for the visualization of lung framework changes were above the level of statistical significance. However, almost half of all HD-IR datasets have an unfamiliar coarse pixel structure. This subjective impression can be objectively confirmed on the basis of histograms of the noise power spectrum.

Despite good image quality evaluations, limitations of the present study in addition to the unfamiliar image appearance and the longer reconstruction time compared to that of FBP must be taken into consideration. These relate in particular to the need to optimize the relationship between dose reduction and the effectiveness of the iterations. The software version we used does not allow deviation from the 5 predefined iteration loops. Additional iterations could result in a further reduction of the image noise while fewer iterations could result in a less unfamiliar image appearance. In the present study design only equal splitting of the tube current to the two X-ray tubes was examined. Step-wise deviations with unequal parts would also be conceivable here. The diagnostic evaluation in the present study was performed purely retrospectively. The effect on clinical evaluation and possible treatment decisions could not be evaluated. The patients were also not selected according to indication and pathologies to be expected. The percentage of inflammatory lesions, for example, was relatively low. The heterogeneous entity of the pathological lesions could differ greatly in a different collective. In addition, a bias regarding lesion evaluation could not be completely ruled out in the evaluation by two separate radiologists with different levels of professional experience. The latter limitations could be responsible for the minor difference in the results compared to the study populations and radiologists of Hwang et al.

The present image quality results achieved with iterative reconstructions of generated computed tomography scans of the thorax at a radiation dose of only $50 \%$ compared to a conventional examination protocol provide the foundation for additional studies for optimizing dose reduction using iterative reconstruction algorithms and their prospective application in the clinical routine.

\section{References}

1 Cohnen M, Poll LJ, Puettmann C et al. Effective doses in standard protocols for multi-slice CT scanning. Eur Radiol 2003; 13: 1148-1153

2 Brix G, Nagel HD, Stamm G et al. Radiation exposure in multi-slice versus single-slice spiral CT: results of a nationwide survey. Eur Radiol 2003; 13: 1979-1991
3 Harrieder A, Geyer LL, Korner M et al. Evaluation der Strahlendosis bei Polytrauma-CT-Untersuchungen eines 64-Zeilen-CT im Vergleich zur 4-Zeilen-CT. Fortschr Röntgenstr 2012; 184: 443 - 449

4 Agency HP, Centre for Radiation CaEH, Division RP. European Commission Radiation Protection $\mathrm{N}^{\circ} 154$, European Guidance on Estimating Population Doses from Medical X-Ray Procedures, Annex 1 - DD Report 1 Review of recent National Surveys of Population Exposure from Medical X-rays in Europe. Chilton, Didcot, Oxfordshire: 2008, In:

5 Widmann G, Fasser M, Schullian $P$ et al. Substantial dose reduction in modern multi-slice spiral computed tomography (MSCT)-guided craniofacial and skull base surgery. Fortschr Röntgenstr 2012; 184: $136-142$

6 Danova D, Keil B, Kastner B et al. Reduction of uterus dose in clinical thoracic computed tomography. Fortschr Röntgenstr 2010; 182: $1091-1096$

7 Hidajat N, Schroder RJ, Vogl T et al. Effektivität der Bleiabdeckung zur Dosisreduktion beim Patienten in der Computertomographie. Fortschr Röntgenstr 1996; 165: 462 - 465

8 Wang J, Duan X, Christner JA et al. Bismuth shielding, organ-based tube current modulation, and global reduction of tube current for dose reduction to the eye at head CT. Radiology 2012; 262: 191 -198

9 Gosch D, Stumpp P, Kahn T et al. Performance of an automatic dose control system for CT: anthropomorphic phantom studies. Fortschr Röntgenstr 2011; 183: 154-162

10 Greess $\mathrm{H}$, Wolf H, Baum U et al. Dosisreduktion in der Computertomographie durch anatomieorientierte schwächungsbasierte Röhrenstromregelung: Erste klinische Ergebnisse. Fortschr Röntgenstr 1999; 170: $246-250$

11 Deak PD, Langner O, Lell $M$ et al. Effects of adaptive section collimation on patient radiation dose in multisection spiral CT. Radiology 2009; 252: $140-147$

12 Honda $O$, Yanagawa $M$, Inoue A et al. Image quality of multiplanar reconstruction of pulmonary $\mathrm{CT}$ scans using adaptive statistical iterative reconstruction. Br J Radiol 2011; 84: 335-341

13 Pontana F, Pagniez J, Flohr T et al. Chest computed tomography using iterative reconstruction vs filtered back projection (Part 1): Evaluation of image noise reduction in 32 patients. Eur Radiol 2011; 21: 627-635

14 Pontana F, Duhamel A, Pagniez J et al. Chest computed tomography using iterative reconstruction vs filtered back projection (Part 2): image quality of low-dose CT examinations in 80 patients. Eur Radiol 2011; 21: 636-643

$15 \mathrm{Hu} X \mathrm{XH}$, Ding XF, Wu RZ et al. Radiation dose of non-enhanced chest CT can be reduced $40 \%$ by using iterative reconstruction in image space. Clin Radiol 2011; 66: 1023-1029

16 Mueck FG, Michael L, Deak $Z$ et al. Upgrade to lterative Image Reconstruction (IR) in MDCT lmaging: A Clinical Study for Detailed Parameter Optimization Beyond Vendor Recommendations Using the Adaptive Statistical lterative Reconstruction Environment (ASIR) Part2: The Chest. Fortschr Röntgenstr 2013; DOI: 10.1055/s-00331335152

17 Deak PD, Smal Y, Kalender WA. Multisection CT protocols: sex- and age-specific conversion factors used to determine effective dose from dose-length product. Radiology 2010; 257: 158-166

18 Bongartz GGS, Jurik AG, Leonardi M et al. European Guidelines on Quality Criteria for Computed Tomography. Danish Society of Radiology 1998, www.drs.dk/guidelines/ct/quality/

19 Julious SA. Sample sizes for clinical trials with normal data. Stat Med 2004; 23: 1921 - 1986

20 Schaich E, Hamerle A. Verteilungsfreie statistische Prüfverfahren. Berlin: Springer; 1984

21 Conover WJ. Practical nonparametric statistics. New York: Wiley; 1980

22 Landis JR, Koch GG. The measurement of observer agreement for categorical data. Biometrics 1977; 33: 159-174

23 Ghetti $C$, Ortenzia O, Serreli G. CT iterative reconstruction in image space: a phantom study. Physica medica 2012; 28: 161 - 165

24 Morsbach F, Desbiolles L, Plass A et al. Stenosis quantification in coronary CT angiography: impact of an integrated circuit detector with iterative reconstruction. Invest Radiol 2013; 48: 32-40

25 Mieville FA, Berteloot L, Grandjean A et al. Model-based iterative reconstruction in pediatric chest $\mathrm{CT}$ : assessment of image quality in a prospective study of children with cystic fibrosis. Pediatric radiology 2013; 43: 558- 567

26 Ebersberger $U$, Tricarico F, Schoepf UJ et al. CT evaluation of coronary artery stents with iterative image reconstruction: improvements in im- 
age quality and potential for radiation dose reduction. Eur Radiol 2013; 23: $125-132$

27 May MS, Wust W, Brand M et al. Dose reduction in abdominal computed tomography: intraindividual comparison of image quality of fulldose standard and half-dose iterative reconstructions with dualsource computed tomography. Invest Radiol 2011; 46: 465 - 470

28 Prakash P, Kalra MK, Ackman JB et al. Diffuse lung disease: CT of the chest with adaptive statistical iterative reconstruction technique. Radiology 2010; 256: 261 - 269

29 Mueck FG, Korner M, Scherr MK et al. Upgrade to iterative image reconstruction (IR) in abdominal MDCT imaging: a clinical study for detailed parameter optimization beyond vendor recommendations using the adaptive statistical iterative reconstruction environment (ASIR). Fortschr Röntgenstr 2012; 184: 229-238

30 Hwang HJ, Seo JB, Lee JS et al. Radiation dose reduction of chest CT with iterative reconstruction in image space - Part I: studies on image quality using dual source CT. Korean J Radiol 2012; 13: 711 - 719

31 Hwang HJ, Seo JB, Lee JS et al. Radiation dose reduction of chest CT with iterative reconstruction in image space - Part II: assessment of radiologists' preferences using dual source CT. Korean J Radiol 2012; 13 : $720-727$ 